\title{
PRÁTICA DE ENFERMAGEM INTERCULTURAL: REVISÃO INTEGRATIVA
}

\section{Eduardo Marques Machado1; Claudia Maria Rivas Ferrony²; Munah Najeh Ahmad Maruf $^{3}$; Liliane Alves Pereira ${ }^{4}$}

\section{RESUMO}

Objetiva-se: identificar a presença do cuidado intercultural em enfermagem, bem como a existência de lacunas do mesmo. Método: trata-se de uma revisão integrativa de literatura. Realizou-se o levantamento de artigos conforme os Descritores em Ciências da Saúde (Decs): Enfermagem; Interculturalidade, com o operador booleano AND. O levantamento dos materiais ocorreu entre agosto e setembro de 2021, nas seguintes bases de dados: Bases de Dados de Enfermagem, Literatura Latino-Americana e do Caribe em Ciências da Saúde e Scientific Eletronic Library Online. Resultados: foram selecionados quatro estudos, onde se identificou fatores norteadores e que contribuem para o exercicio do cuidado intercultural, tendo maior prevalencia a comunicação e o respeito. Conclusão: A presente revisão evidência pontos cruciais para a implementação do cuidado intercultural em enfermagem. Devido a pequena quantidade de estudos, se dificulta a analise acerca do cuidado intercultural, a amostra se torna menor a nível nacional, sem poder mensurar a realidade brasileira.

Palavras-chave: Cuidado; Enfermagem; Interculturalidade.

Eixo Temático: Atenção Integral e Promoção à Saúde.

\section{INTRODUÇÃO}

Segundo Osorio \& López (2008), na década de 1980, a Organização Mundial da Saúde, incorporou o reconhecimento a cultura e a diversidade cultural ao cuidado em saúde. Esse aspecto está presente no dia a dia, se tornando uma dimensão fundamental nas políticas e programas em saúde.

As práticas de enfermagem, são entendidas como um conjunto de saberes técnico-científicos. A atenção integral a saúde é influenciada por fatores como

\footnotetext{
${ }^{1}$ Apresentador. Graduando em enfermagem - UFN. eduardomarques051@gmail.com

${ }^{2}$ Graduanda em enfermagem - UFN. claudiamfrivas@gmail.com

${ }^{3}$ Graduanda em enfermagem - UFN. munahsaleh1999@gmail.com

${ }^{4}$ Orientadora. Professora do Curso de Enfermagem - UFN. liliane.pereira@ufn.edu.br
} 
processos culturais, históricos, sociais, políticos e econômicos. Para isso, ao se tratar de um cuidado que se relacione com o bem-estar do indivíduo, é necessária atenção para os fatores socioculturais, espirituais, econômicos, entre outros (RAYMUNDO, 2013).

A teoria do cuidado cultural, foi a primeira tentativa de destacar a necessidade da competência cultural por enfermeiros. Onde explica que os enfermeiros devem ter conhecimento de diferentes culturas, com 0 intuito de prestar cuidado a diferentes etnias. É impossível deter conhecimento sobre todas as perspectivas culturais, mas se torna relevante adquirir conhecimento e competência em comunicação intercultural para o cuidado singular (LEININGER, 2002).

O enfoque no que tange a singularidade do paciente tem a necessidade de uma mudança de paradigmas para um modelo biopsicossociocultural, onde seja capaz atender as demandas de cada indivíduo. Esse modelo de atenção proporciona que o cuidado não esteja vinculado apenas onde existe um ponto de desequilíbrio em saúde. Com isso, o paciente tem maior autonomia para a busca da atenção à saúde, conforme seus significados e crenças (RAYMUNDO, 2013). Assim, surge um modelo de cuidado que utiliza a interculturalidade como ferramenta, para a busca da atenção integral a saúde do paciente.

A interculturalidade pode ser considerada como um processo de construção social, estimulada pela tensão cultural. Essa ocorre quando existem conflitos interculturais estimulados pela oposição de valores, pertença religiosa, posição política e incompatibilidade de interesses. Desta forma, a construção intercultural permeia a convivência, a inclusão e a diversidade (COSTA; SANTOS, 2017).

A interculturalidade se fundamenta em promover o diálogo a partir das perspectivas culturais de cada interlocutor. Para isso, conhecer a cultura do outro e reconhecer a própria, se torna primordial. Diante disso, se é possível estabelecer relações éticas e realizar a neutralização de preconceitos (RAYMUNDO, 2013).

O cuidado intercultural propõe que a forma de cuidado seja singular, sem que haja uma cultura superior a outra. Na existência de conflitos, a interculturalidade é colocada na vanguarda, onde é a capaz de promover um diálogo, ampliando os horizontes e assim enriquecendo os laços com respeito às diferenças culturais, 
estabelecendo uma ponte e uma rede intercultural, diminuindo os preconceitos e conflitos entre pessoas pelas suas diferenças, possibilitando elos de confiança entre os envolvidos (WEISSMANN, 2018).

O presente trabalho tem como objetivo identificar a presença do cuidado intercultural em enfermagem, bem como a existência de lacunas do mesmo. Assim, elegeu-se a seguinte questão norteadora: "Como a interculturalidade está presente na prática de enfermagem?".

\section{METODOLOGIA}

Trata-se de uma revisão integrativa de literatura. Para a realização da revisão foram executadas as seguintes etapas metodológicas: definição da temática, definição da questão norteadora, busca e seleção de estudos primários, extração dos dados dos estudos primários, avaliação crítica dos artigos, síntese dos resultados da revisão e apresentação da revisão (MENDES; SILVEIRA; GALVÃO, 2019).

Realizou-se o levantamento de artigos conforme os Descritores em Ciências da Saúde (Decs): Enfermagem; Interculturalidade, com o operador booleano AND. O levantamento dos materiais ocorreu entre agosto e setembro de 2021, nas seguintes bases de dados: Bases de Dados de Enfermagem (BDENF), Literatura LatinoAmericana e do Caribe em Ciências da Saúde (LILACS) e Scientific Eletronic Library Online (SciELO), sendo BDENF e LILACS, realizada a busca na Biblioteca Virtual em Saúde (BVS).

Os critérios de elegibilidade foram: textos completos disponíveis na íntegra que continham os descritores selecionados no título ou no resumo, publicados entre 2011 e 2021. Os critérios de exclusão foram: estudos de relato de caso, relato breve, estudos de revisão, editoriais e artigos que não atenderam o objetivo proposto. $O$ recorte temporal escolhido é referente a 10 anos. Durante a busca, foram selecionados os filtros de base de dados que correspondem a BVS, texto completo, idioma e intervalo de tempo.

As etapas de busca dos materiais estarão dispostas nos resultados da pesquisa, por meio de um quadro. Da mesma, também estarão disponíveis os 
estudos encontrados durante a busca, conforme referência, objetivo e principais resultados. A busca e síntese dos resultados foi feita por dois revisores independentes.

\section{RESULTADOS}

Fluxograma 1- artigos selecionados através da base de dados entre agosto e setembro de 2021. Santa Maria/ RS, 2021.
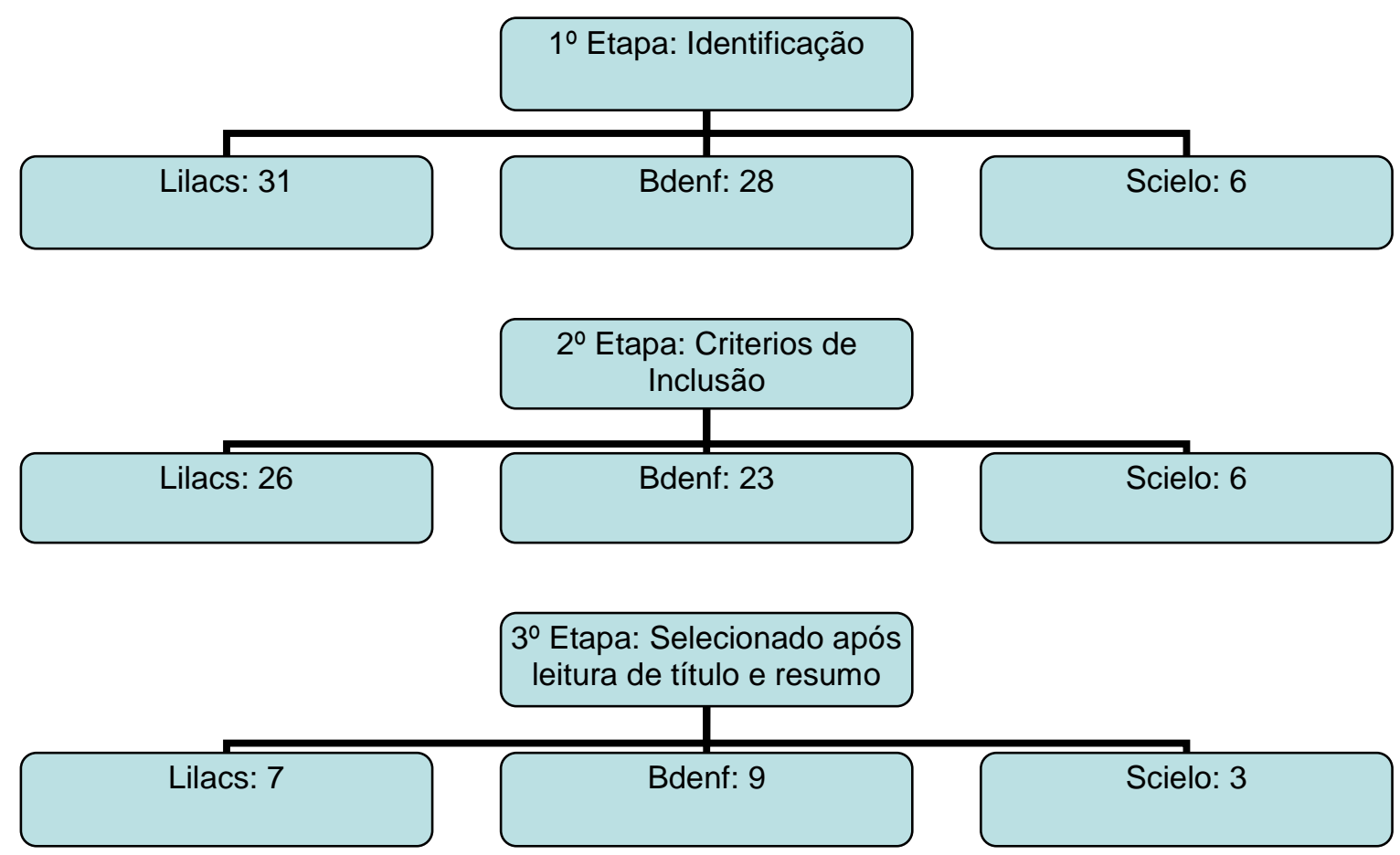

\section{Excluídos por Duplicidade: 9}

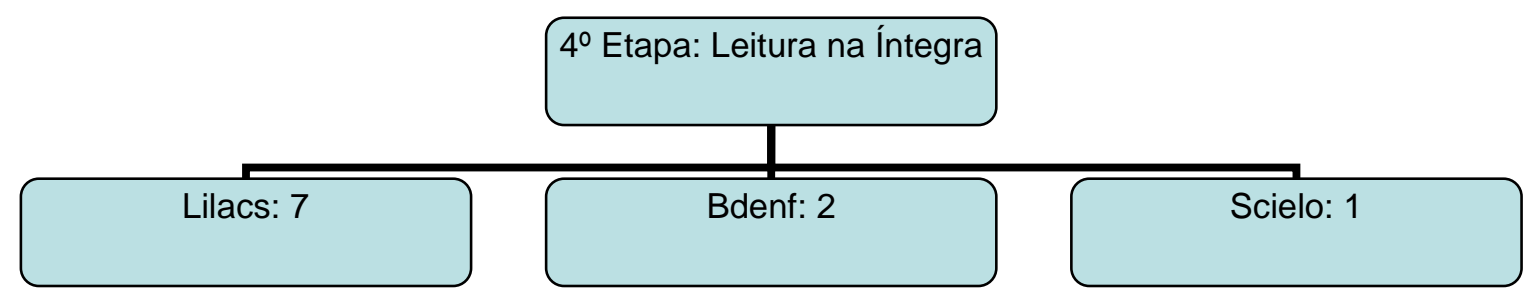

Excluídos por não responder à questão norteadora: 6 


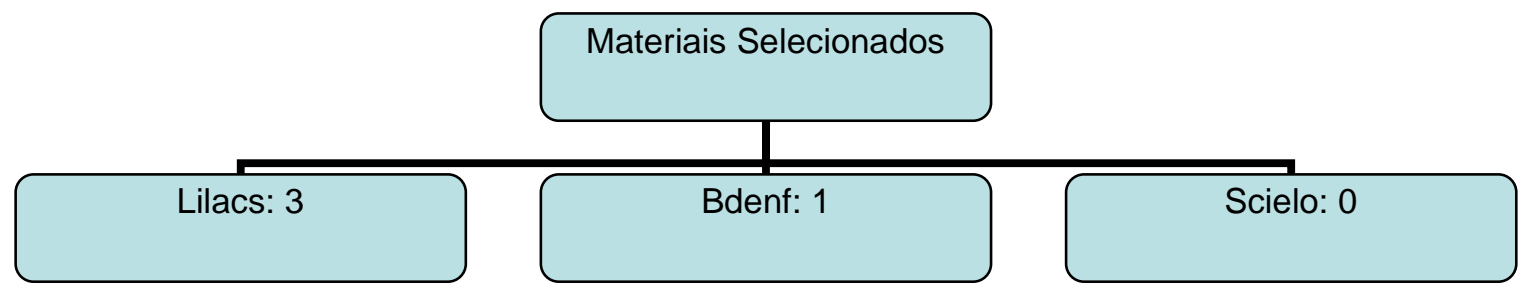

Fonte: Autores.

No quadro a seguir estão disponíveis os estudos selecionados para compor a presente revisão integrativa, sendo esse em um número total de quatro artigos, sendo três materiais na base LILACS e um na base BDENF. Quanto à caracterização dos mesmos observou-se que três artigos são internacionais e um nacional.

Quadro 1 - Síntese dos artigos, conforme identificação, título, autores, ano, objetivo, método e conclusão. Santa Maria/ RS, 2021.

\begin{tabular}{|c|c|c|c|c|c|}
\hline $\begin{array}{c}\text { Base } \\
\text { de } \\
\text { dados }\end{array}$ & ID / Título & $\begin{array}{c}\text { autores/ } \\
\text { ano }\end{array}$ & Objetivo & Método & Conclusão \\
\hline \multirow[t]{2}{*}{ Lilacs } & $\begin{array}{l}\text { L1/ } \\
\text { Transcultur } \\
\text { alidade: o } \\
\text { enfermeiro } \\
\text { com } \\
\text { competênci } \\
\text { a cultural }\end{array}$ & $\begin{array}{c}\text { Vilelas, } \\
\text { Janeiro/ } \\
2012 .\end{array}$ & $\begin{array}{c}\text { Discutir as } \\
\text { mudanças } \\
\text { importantes para a } \\
\text { promoção de uma } \\
\text { enfermagem } \\
\text { transcultural. }\end{array}$ & $\begin{array}{l}\text { Trata-se de } \\
\text { um estudo } \\
\text { teórico } \\
\text { reflexivo. }\end{array}$ & $\begin{array}{c}\text { Deve-se incutir nos } \\
\text { estudantes de enfermagem } \\
\text { o interesse pela diversidade } \\
\text { cultural diferenças de } \\
\text { valores, crenças, costumes } \\
\text { relativos à saúde, pois } \\
\text { ajudará a enfermagem a } \\
\text { assegurar a competência } \\
\text { cultural. }\end{array}$ \\
\hline & $\begin{array}{l}\quad \text { L2/ } \\
\text { Dimension } \\
\text { es de la } \\
\text { competenci } \\
\text { a cultural } \\
\text { en } \\
\text { enfermería } \\
\text { y prácticas } \\
\text { tradicionale } \\
\text { s para el } \\
\text { cuidado de } \\
\text { la infancia }\end{array}$ & $\begin{array}{c}\text { Díaz U, } \\
\text { López T/ } \\
2015 .\end{array}$ & $\begin{array}{c}\text { Identificar } \\
\text { expressões das } \\
\text { dimensões da } \\
\text { competência } \\
\text { cultural em } \\
\text { enfermeiras, foi } \\
\text { desenvolvida uma } \\
\text { pesquisa qualitativa } \\
\text { fenomenológica } \\
\text { com a } \\
\text { participação }\end{array}$ & $\begin{array}{l}\text { Pesquisa } \\
\text { qualitativa } \\
\text { de tipo } \\
\text { fenomenoló } \\
\text { gico. }\end{array}$ & $\begin{array}{c}\text { É possível perceber que o } \\
\text { desenvolvimento da } \\
\text { competência cultural nos } \\
\text { profissionais de } \\
\text { enfermagem está associado } \\
\text { a suas próprias crenças } \\
\text { culturais e na experiência } \\
\text { proveniente do exercício } \\
\text { profissional. Há conflitos } \\
\text { identificáveis nas diferentes } \\
\text { competências culturais }\end{array}$ \\
\hline
\end{tabular}




\begin{tabular}{|c|c|c|c|c|c|}
\hline & & & $\begin{array}{l}\text { voluntária de onze } \\
\text { enfermeiras, cuja } \\
\text { experiência } \\
\text { principal foi o } \\
\text { cuidado de crianças } \\
\text { em diferentes } \\
\text { campos. }\end{array}$ & & $\begin{array}{l}\text { entre o fazer, o ser e o } \\
\text { dever tanto no exercício } \\
\text { como na aplicação de }\end{array}$ \\
\hline & $\begin{array}{l}\text { L3/ } \\
\text { Competênc } \\
\text { ia cultural } \\
\text { de } \\
\text { enfermeira } \\
\text { s em saúde } \\
\text { pública } \\
\text { com } \\
\text { população } \\
\text { indígena }\end{array}$ & $\begin{array}{l}\text { Castillo, } \\
\text { López- } \\
\text { Díaz/ } \\
2019 .\end{array}$ & $\begin{array}{c}\text { Identificar } \\
\text { elementos de } \\
\text { competência } \\
\text { cultural (cc) das } \\
\text { enfermeiras da } \\
\text { saúde pública (esp) } \\
\text { quando atendem à } \\
\text { população indígena } \\
\text { emberá-chamí. }\end{array}$ & $\begin{array}{c}\text { Etnografia } \\
\text { interpretativ } \\
\text { a com } \\
\text { observação } \\
\text { participante } \\
\text { e } \\
\text { entrevistas } \\
\text { em } \\
\text { profundidad } \\
\text { e com dez } \\
\text { colaborador } \\
\text { es. }\end{array}$ & $\begin{array}{l}\text { O estudo oferece } \\
\text { evidências de como o CC } \\
\text { na saúde é um processo } \\
\text { amplo, não só de PES com } \\
\text { os indígenas, mas também } \\
\text { de sua adoção por sistemas } \\
\text { e instituições com políticas, } \\
\text { planos e programas } \\
\text { apoiados por culturas } \\
\text { indígenas. }\end{array}$ \\
\hline BDenf & $\begin{array}{l}\text { B1/ } \\
\text { Percepção } \\
\text { dos } \\
\text { cuidados } \\
\text { de } \\
\text { enfermage } \\
\text { m por } \\
\text { indígenas } \\
\text { Embera }\end{array}$ & $\begin{array}{c}\text { Castillo, } \\
\text { López- } \\
\text { Díaz/ } \\
2019 .\end{array}$ & $\begin{array}{c}\text { Interpretar a } \\
\text { percepção dos } \\
\text { indígenas Embera } \\
\text { Chami frente à } \\
\text { competência } \\
\text { cultural do } \\
\text { enfermeiro de } \\
\text { saúde pública. }\end{array}$ & $\begin{array}{c}\text { Etnografia } \\
\text { interpretativ } \\
\text { a com } \\
\text { observação } \\
\text { participante } \\
\text { e } \\
\text { entrevistas } \\
\text { etnográfica } \\
\text { s. }\end{array}$ & $\begin{array}{c}\text { Os indígenas sentem } \\
\text { satisfação e compromisso } \\
\text { em traduzir as } \\
\text { recomendações oferecidas } \\
\text { pelo enfermeiro, } \\
\text { principalmente quando o } \\
\text { cuidado do profissional é } \\
\text { feito de forma culturalmente } \\
\text { competente, envolvendo o } \\
\text { contexto dos indígenas. }\end{array}$ \\
\hline
\end{tabular}

Fonte: Autores.

O artigo (L1), aponta determinados pontos referentes a competência cultural, onde esses podem variar a compreensão em relação ao enfermeiro e aquele que recebe o cuidado, sendo a comunicação, o contato com os olhos, o toque, o silêncio, o espaço e distância e as crenças em saúde. Assim, sugere três etapas para a promoção da competência cultural, essas: Adotar atitudes para promover a transculturalidade nos cuidados de enfermagem; desenvolver a consciência das diferenças culturais; e realizar uma avaliação cultural. Salienta-se ainda que para esse cuidado é necessário ética e educação em enfermagem transcultural. 
O próximo artigo (L2), se refere a competência cultural de enfermagem no cuidado durante a infância. Para isso, são identificadas seis dimensões: consciência cultural, conhecimento cultural, habilidade cultural, desejo cultural, sensibilidade cultural e encontro cultural. Tratam respectivamente de reconhecer as características culturais particulares; interesse pela aprendizagem da cultura para compreender o próximo; capacidade de atuar a partir da compreensão do outro; interesse por entender e ser empático com outro, reconhecer e aceitar as diferenças; estabelecimento de relações interpessoais baseadas no respeito; e capacidade de interesse e compreensão de aspectos que promovam inclusão, tolerância e dialogo de saberes. O desenvolvimento da competência cultural, também está relacionada as próprias crenças e experiências geradas em seu exercício profissional.

O terceiro artigo (L3), discute os elementos necessários para competência cultural de enfermeiras, que prestam assistência a uma comunidade indígena. $\mathrm{O}$ primeiro aspecto se trata da união de saberes ocidentais e tradicionais em relação a saúde. Por conseguinte, o respeito e a importância da fusão de saberes, onde o cuidado cultural está dirigido as particularidades. A abordagem estende-se a formulação de políticas públicas que proporcionem um enfoque culturalmente competente, onde a interculturalidade se mostre um princípio fundamental. Por último, os aspectos verbais e não verbais, a comunicação se dá em espanhol, mas os profissionais de enfermagem também devem compreender a linguagem coloquial dos indígenas. A comunicação se mostra como um processo unificador no cuidado intercultural.

O último artigo (B1), trata da competência cultural de enfermeiros durante o cuidado a indígenas na Colômbia. O cuidado é percebido no conjunto de ações institucionais e profissionais associadas a: rapidez no acesso aos serviços, apoio financeiro para tratamentos, redução dos tempos de espera no atendimento, integração dos saberes tradicionais e ocidentais. Dentro de suas crenças consideram que os dois saberes têm lugar e aceitação de forma natural. Questões relacionadas à alimentação balanceada são difíceis de atender a esse grupo populacional. Desta forma, se torna importante a compreensão e adaptação do que 
essa cultura entende por saúde, para assim prestar assistência com competência cultural.

\section{DISCUSSÕES}

Diante da premissa de que o cuidado intercultural, constitui parte do processo de sistematização da assistência de enfermagem de modo integral, torna-se pertinente discutir o mesmo.

Ramos (2013), identifica que a comunicação deve estar adaptada ao nível cultural/educacional, às necessidades individuais, emocionais, sociais, culturais e linguísticas do utente/doente. Onde a compreensão daquele que recebe os cuidados é influenciada pelas suas crenças, tradições, confiança e o respeito as diferenças. A dificuldade na comunicação pode conduzir para uma atitude técnica e impessoal, dificultando a compreensão social e aumentando a vulnerabilidade.

A comunicação permite relações, troca de experiencias de vida e compartilhamento de saberes, as relações interpessoais de confiança e respeito proporcionam bem-estar (PEREIRA; MACHADO; TOLFO, 2020). Nessa perspectiva, a comunicação clara, se faz necessária para a construção de uma troca intercultural, assim proporcionando ótimo ambiente tanto para usuário e profissional.

Em relação ao cuidado intercultural a comunidade indígena, o Brasil, tem um subsistema a de Atenção à Saúde Indígena, com a Política Nacional de Atenção à Saúde dos Povos Indígenas (PNASPI). A mesma orienta a uma perspectiva intercultural e indigenista, tais como a articulação entre saberes, respeito de práticas e valores do sistema tradicional e a consideração e reconhecimento da diversidade. Se tem uma lacuna no que diz respeito à ausência de investimento em processos de formação diferenciados, com base no conhecimento tradicional do contexto local e competências relacionais e comunicativas interculturais (PEDRANA et al., 2018).

Com base na competência cultural no ato de cuidar, Estevan \& Ruíz (2017), referem que aplicar o modelo de Purnell, permite identificar a competência cultural dos profissionais de enfermagem. Constituindo uma abordagem etnográfica, oferecendo cuidados de qualidade e culturalmente congruentes. 
O enfermeiro com competências culturais tem maior facilidade de compreender os modos saúde como as práticas adotadas variam de indivíduo para indivíduo. A mesma tem algumas dimensões, sendo: melhorar a sensibilidade dos profissionais de saúde às crenças culturais, práticas, expectativas e origens de seus pacientes e suas comunidades; melhorar 0 acesso aos cuidados de saúde, eliminando as barreiras estruturais aos cuidados de saúde de qualidade; e reduzir as barreiras organizacionais, como o pequeno número de profissionais de saúde, administradores e elaboradores de políticas (DAMASCENO; SILVA, 2018).

A prática de enfermagem em saúde culturalmente segura, se caracteriza pelo respeito. Acontece quando o profissional de saúde desenvolve a sensibilidade cultural, sendo capaz de refletir sobre sua própria cultura e a influência que a mesma exerce em sua prática (GURM; CHEEMA, 2013).

A interculturalidade é compreendida pelos profissionais de saúde, como variedade, quer seja de saberes, práticas ou culturas diferenciadas. Assim, a interculturalidade se torna a integração entre dois sistemas de conhecimentos: o ocidental e o tradicional próprio dos usuários. Desta forma, implica em um harmonioso intercambio de saberes em uma relação interpessoal (ARIAS-MURCIA; PENNA, 2021).

A interculturalidade junto aos preceitos bioéticos conduzem o usuário e profissional a uma relação integral. Essa é capaz de produzir respeito, confiança, atenção, cuidado, vida. Com isso, não se coloca superioridade, mas identificação de suas diferenças, na busca de uma construção de sociabilidade (MACHADO; FLORES; PEREIRA, 2019).

Para que o cuidado de enfermagem tenha maior impacto positivo na vida dos pacientes, se torna pertinente respeitar as diferenças culturais como costumes, crenças, rituais, hábitos de vida e valores (LIMA et al., 2016). Assim, o enfermeiro deve compreender todos fatores intrínsecos e extrínsecos que estão relacionados a saúde do paciente como seu aspecto cultural.

Coutinho et al., (2017), ao fazer referência a teorista Madeleine Leininger, traz que a enfermagem ao assumir a centralidade do cuidado, se torna uma profissão 


\section{QuFN}

essencialmente intercultural. Visto que o indivíduo é detentor de uma forma muito particular de ver o mundo, em função das suas crenças, valores, costumes e práticas culturais.

\section{CONCLUSÃO}

A presente revisão evidência pontos cruciais para a implementação do cuidado intercultural em enfermagem. Tendo destaque para a comunicação, as competências que o enfermeiro necessita para o cuidado cultural, a união de saberes e práticas em saúde, crenças, tradições e valores, o respeito e a formulação de políticas que abordem a compreensão cultural.

Ademais, se torna pertinente maiores estudos acerca da temática e aprofundamento dos aspectos que envolver o cuidado intercultural em enfermagem. Visto que, o mesmo está presente em toda a prática de enfermagem e reconhecendo o contexto dos indivíduos.

\section{Limitações do Estudo}

Devido a pequena quantidade de estudos, se dificulta a analise acerca do cuidado intercultural, a amostra se torna menor a nível nacional, sem poder mensurar a realidade brasileira. Os estudos ainda mostram que o cuidado intercultural está muito ligado a população indígena e não é aplicado e avaliado a nível de diferenças micro sociais.

\section{REFERÊNCIAS}

ARIAS-MURCIA, S. E.; PENNA, C. M. M. A interculturalidade no cotidiano da atenção primária à saúde: $\mathrm{O}$ caso do modelo de saúde em Guainía, Colômbia. Ciência \& Saúde Coletiva, v. 26, (Supl. 2), p. 3683-3692, Rio de Janeiro, 2021.

COSTA, P. E.; SANTOS, J. A. Diversidade Cultural, Convivência, Conflito E Mediação. Revista da UIIPS - Unidade de Investigação do Instituto Politécnico de Santarém, v. 5, n. 4, Santarém, 2017. 
COUTINHO, E et al. O cuidado cultural na trajetória da enfermagem transcultural e competência cultural. Atas do 60 Congresso Ibero-Americano em Investigação Qualitativa, vol. 2, Salamanca, 2017.

DAMASCENO, R. F.; SILVA, P. L. N. Competência cultural na atenção primária: algumas considerações. J Manag Prim Health Care [Internet]., v. 9, Uberlândia, 2018.

ESTEVAN, M. D. G.; RUÍZ, M. C. S. La aplicación del modelo de competencia cultural en laexperiencia del cuidado en profesionalesde Enfermería de Atención Primaria. Aten Primaria., v. 49, n. 9, p. 549-55, España 2017.

GURM, B. K.; CHEEMA, J. Cultural Safety Assessment of an Urban Canadian Hospital. Journal of Cultural Diversity., v. 20, p. 177-83, Lisle, 2013.

LEININGER, M. Culture care theory: a major contribution to advance transcultural nursing knowledge and practices. J Trans Nurs., v. 13, n. 3, p. 189, 2002.

LIMA, M. R. A. et al. Atuação de enfermeiros sobre práticas de cuidados afrodescendentes e indígenas. Revista Brasileira de Enfermagem, v. 69, n. 5, p. 840-846, Brasília, 2016.

MACHADO, E.M.; FlORES, A. N. D.; PEREIRA, L. A. A Interculturalidade Em Enfermagem: Um Olhar Bioético. In: Jornada Internacional de Enfermagem, 2019, Santa Maria. 6a Jornada Internacional De Enfermagem E 4ํㅗㄴ Seminário Em Saúde Materno Infantil: Sistematização do processo de cuidado em saúde. Santa Maria, Rio Grande do Sul, 2019.

MENDES, K. D. S.; SILVEIRA, R. C. C. P.; GALVÃO, C. M. Uso de gerenciador de referências bibliográficas na seleção dos estudos primários em revisão integrativa. Texto contexto - enferm, v. 28, e20170204, Florianópolis, 2019. 
OSORIO, M.; LÓPEZ, A. Competencia cultural en salud: necesidad emergente en un mundo globalizado. Index Enferm [Internet], v. 17, n. 4, p. 266-7, Granada, 2008.

PEDRANA, L. et al. Análise crítica da interculturalidade na Política Nacional de Atenção às Populações Indígenas no Brasil. Rev Panam Salud Publica, v. 42, Washington, 2018.

PEREIRA, L. A.; MACHADO, M. E.; TOLFO, K. Relação professor e estudante em tempos de pandemia. Rev. Franc. Edu., v. 4, p. 14-24, Santa Maria, 2020.

RAMOS, N. Cuidados de saúde e comunicação na sociedade multicultural: discutindo interculturalidade (s), práticas e políticas em saúde. Revista Inter-Legere, v. 1, n. 11, p. 30-51, Natal, 2013.

RAYMUNDO, M. M. Interculturalidade e a conjunção de saberes que congregam a atenção em saúde. Rev. bioét. (Impr.)., vol. 21, n. 2, p. 218-25, Brasilia, 2013.

WEISSMANN, L. Multiculturalidade, transculturalidade, interculturalidade. Constr. psicopedag., v. 26, n. 27, São Paulo, 2018. 